
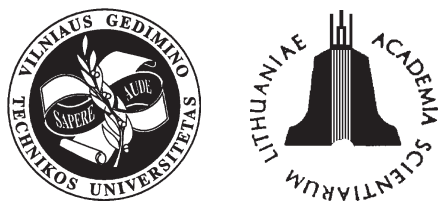

ISSN 1648-4142 TRANSPORT

http:/www.vtu.lt/english/editions

TRANSPORT - 2005, Vol XX, No 1, 23-31

\title{
MODELLING OF ELECTROMECHANICAL DRIVE SYSTEMS WITH THE USE OF MODAL CONDENSATION
}

\author{
Damian Gąsiorek, Arkadiusz Męžyk, Eugeniusz Świtoński \\ Dept of Applied Mechanics, Silesian University of Technology, Gliwice, Poland, \\ tel.: (0 32) 237-12-87, fax.: (0-32) 237-13-09.E-mail: Damian.Gasiorek@polsl.pl \\ Received 2004-01-13; accepted 2004-12-03
}

\begin{abstract}
This paper presents a method of developing a dynamic model enabling the study of the effect of the flexibility of the housing on dynamic phenomena in electromechanical drive systems. The research was performed on the basis of an electromechanical model with feedback between the mechanical subsystem (toothed gear with housing) and the electrical subsystem using a software package developed by the author in MATLAB environment.
\end{abstract}

Keywords: modal condensation, electromechanical systems.

\section{Introduction}

Very often, due to adverse conditions prevailing during the operation of machines, high cost and the need to use specialised measuring equipment in experimental tests the research is restricted to model testing. Usually experimental testing is merely the starting point for further theoretical considerations. Experiments are conducted under specified operating conditions and the results obtained are valid for these conditions. Model testing is useful, for instance, in the analysis, synthesis and optimisation of high power transmission systems. In theoretical research mathematical models are used which to a large extent represent the real systems and the results obtained enable quantitative and qualitative assessment of dynamic phenomena. One of the problems in the process of mathematical modelling is a large number of parameters that describe the real object. Therefore, the assumptions made must be simplified and the most important parameters must be selected to describe the phenomenon. Quantitative and qualitative assessment of the effect of design features on the dynamic phenomenon being studied is important as these features have an impact on the nature and size of the dynamic forces in kinematic pairs. Maximum permissible size of forces in kinematic pairs can be determined from the time variations obtained with the use of numerical methods.

Machine drives constitute complex and complicated dynamic systems with a large number of degrees of freedom and they require a proper approach to their mathematical description. The dynamic condition of a machine is most often modelled in the form of a complex dynamic system consisting of a number of simple interacting subsystems. An example of such an approach is the modelling of a drive as an electromechanical system with feedback between its electrical part and its mechanical part [1,2]. In this case the electromagnetic system is usually described by Maxwell's laws, while the mechanical system is described by the rules and laws of mechanics.

Systems with many degrees of freedom are modelled to represent large electromechanical systems. Such systems require solving a large number of second order differential equations which results in protracted numerical computations. There are, however, methods that enable reducing the number of degrees of freedom of a system. These reduction methods allow faster determination of the size and forms of the free vibrations of the system studied and make the static and dynamic analysis of the task examined less labour-intensive. Reduction also plays an important role in an experimental modal analysis as mass and stiffness matrices can also be used to compare analytical and experimental models by controlling their orthogonality.

\section{Modelling of electromechanical systems}

High power machines usually operate under dynamically variable loads. Such systems can be broken 
up into an electromagnetic system described by Maxwell's laws and a mechanical system described by the rules and laws of mechanics.

The behaviour of electromechanical systems in transient and resonant states is the most interesting, therefore complex dynamic models comprising mechanical and electromagnetic subsystems are constructed. Such a model should take into account the most important dynamic features of the system as well as the most important external forces acting on it. The model should also take into account the feedback between the electrical system and the mechanical system.

The mechanical part usually consists of gears (modelled in a discrete or discrete-continuous manner). The characteristics of the stiffness of meshing, flexibility of supports, backlash, stiffness of shafts and the housing, etc. are taken into account. A system of ordinary equations is used to describe the mechanical system:

$$
\mathbf{M} \ddot{\mathbf{q}}+(\mathbf{B}+\mathbf{G}) \dot{\mathbf{q}}+\mathbf{K q}=\mathbf{Q},
$$

where $\mathbf{M}, \mathbf{B} \mathbf{G}, \mathbf{K}$ - matrices of inertia, damping, gyroscopic effect and stiffness, $\mathbf{q}-$ vector of generalised coordinates, $\mathbf{Q}$ - vector of generalised forces.

Dynamic equations of motion for an asynchronous machine model can be formulated as follows:

$$
\begin{aligned}
& \frac{d}{d t} \mathbf{L i}+\mathbf{R i}=\mathbf{U}, \\
& M_{e l}=\frac{1}{2} \mathbf{i}^{T} \frac{\partial}{\partial \varphi_{1}} \mathbf{L i},
\end{aligned}
$$

where $\mathbf{L}, \mathbf{R}, \mathbf{i}, \mathbf{U}$ - matrices of inductance, resistance, supply currents and voltages; $\mathrm{M}_{\mathrm{el}}-$ moment of the motor, $\varphi_{1}$ - rotor rotation angle.

Electromechanical coupling is effected by the rotor rotation angle $\mathrm{jl}$ derived from the mechanical system model and by electromagnetic moment Mel derived from the electric motor model. Solution of the above motion equations of the coupled system model enables the determination of its dynamic characteristics.

\subsection{Model of the mechanical system}

The adopted degree of simplification of mechanical models depends on the purpose of research and on the identifiability of the parameters used. This ensues mainly from the complexity of toothed gear trains which constitute dynamic systems with many degrees of freedom. The adopted model should take into account the features of the real object. These features have a significant effect on the studied dynamic phenomena. To a large extent the accuracy of the adopted model depends on the purpose of the study. However, one must bear in mind the problems brought about by the determination of parameters of the adopted model. The first stage in mechanical system modelling is the adoption of a physical model, the subsequent stage is the discrete model and finally there is a mathematical model.

The simplest classification of models found in the literature is the one that differentiates between strongly and weakly nonlinear systems. The former system is one where linearised models can be applied with good approximation. An example of a strongly nonlinear system is a system with play.

Energy dissipation phenomena are difficult to handle when trying to describe a model mathematically and they impose many simplifying assumptions in the modelling process. It is an accepted practice that when systems of a given class are modelled, the damping forces are ignored in the first approximation and in the next step damping matrices are construed in the form of a linear combination of inertia and stiffness matrices:

$$
\mathbf{B}=\alpha_{1} \mathbf{M}+\beta_{1} \mathbf{K},
$$

where $\alpha_{1}, \beta_{1}$ - are scalar coefficients.

This method simplifies considerably the analysis of damped vibrating systems. It is often assumed $\alpha_{1}=0$, while $\beta_{1}$ is determined from material constants and in consequence the damping matrix is proportional to the stiffness matrix.

The simplest model of a gear is a torsional vibrations model, whereas a somewhat more complex model is the transverse and torsional vibrations model. However, selecting one of the models is a difficult problem faced by anyone trying to create a model of a mechanical system.

\section{Modelling of system using the modal condensation method}

In dynamics many a times we encounter the problem of modelling vibrations of a mechanical system composed of many " $\mathrm{j}$ " subsystems interconnected by means of many discrete links (Fig 1). Single subsystems are often replaced with subsystems described by concentrated parameters composed of discrete elements.

Individual subsystems oscillate in a static state of equilibrium with small deflections and then the vibrations can be described with some approximation, while ignoring nonlinear damping forces by means of a system of differential equations with constant coefficients $(3,4)$. Each such subsystem is a discrete linear model with $n_{j}$ degrees of freedom. These simple 


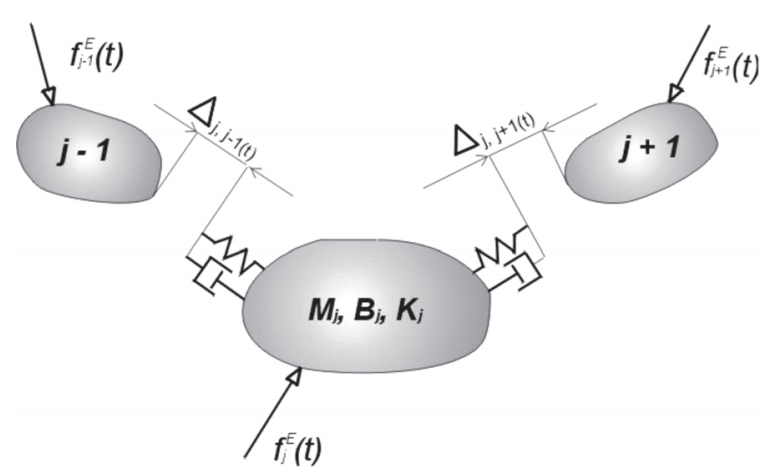

Fig 1. A mechanical system composed of subsystems [5]

subsystems cannot be replaced with a system with averaged parameters and it is necessary to discretise them.

\subsection{Model of a toothed gear train}

A toothed gear train (Fig 2) can be modelled as a system of shafts with mass concentrated in the form of disks at points of gear mounting supported in bearings. Coupling between shafts is effected by meshing between adjacent gears. Shaft discretisation can be done using FEM [6-12] upon dividing the shaft into a finite number of continuous sections of constant diameter. Each shaft with gears mounted on it, couplings, supports at points of bearing mounting constitutes an isolated subsystem.

The rigid finite element method enables creating a model that describes the system with sufficient fidelity in the area of low free vibration frequencies. If, however, analysis of a wide range of frequencies or of higher frequencies is required, then a hybrid method must be applied. In this method gears are treated as solid bodies, whereas shafts, the rigidity of which is several orders lower, are divided into deformable finite beam elements.

The rigid finite element method is applied to

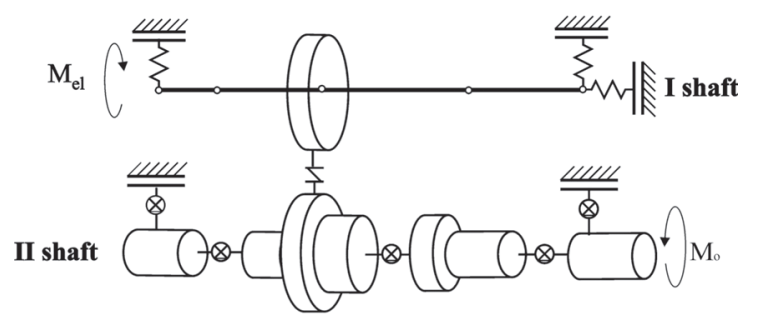

$$
\begin{array}{cl}
\multimap & \text { - beam finite element } \\
-\otimes- & \text { - elasto-dumping element } \\
\frac{1}{+} & \text { - stiffness in gear } \\
\square 0 & \text { - rigid finite element } \\
\mathrm{Mel}_{\mathrm{el}} & \text { - driving moment } \\
\mathrm{M}_{0} & \text { - anti-torque }
\end{array}
$$

Fig 2. Hybrid model of a gear train [2] model discrete fragments, i.e. those with concentrated parameters. On the other hand, for the modelling of continuous elements, i.e. those with mass, elastic and damping properties distributed continuously in space, the finite element method is applied. Elastically damping elements are used for the modelling of the interface between mating gears.

The forces that act upon subsystems include the drive forces transferred from the electric motor, resisting forces on the output, interaction between shafts the latter usually represented by forces between gear teeth or forces acting in the coupling.

\subsection{Model of the housing}

The housing of the gear train is significant from the viewpoint of dynamic properties of the entire mechanical and electrical system. Standard finite elements software is usually used to model the housing. The Ansys software which enables analysing dynamic phenomena in systems modelled was applied to generate the model of a gear housing. One important advantage of this software is its ability to determine the frequency of free vibrations of the housing.

Ansys uses an APDL internal programming language which can be applied to determine the form of free vibrations in nodes and to export them as text files to MatLab software.

A model representing the real object is created on the basis of technical design documents.

Some problems may be encountered when a mathematical model of a structure is created with the use of the finite element method - models with a high number of degrees of freedom are generated $(n>103)$. Such models are too large for further analysis of dynamic properties and optimisation due to hardware and computation time constraints. When modal synthesis is applied to determine dynamic responses of defined structures, a relatively small number of free vibration forms $(\mathrm{m}<100)$ can be taken into account.

It is therefore proper for the housing finely divided into finite elements, when arranging a complete condensed system, to reduce the number of eigenvectors. The reduction of the number of eigenvectors brings about simplification as it enables to disregard many equations without a notable impact on the dynamic analysis results obtained with the use of modal synthesis. These generalised equations of nodes should be retained where connections with the preceding subsystem are defined. In the case of housing these may be the nodes where connections with the bearing are defined.

\subsection{Modelling connections between subsystems}

Modelling of discrete connections between subsystems is done in a global system of equations of the 
system described by a vector of generalised equations $\mathbf{q}(t)=\left[\mathbf{q}_{j}(t)\right]$ of dimension $n=\sum n_{j}$, where $n_{j}$ is the number of degrees of freedom of the individual subsystems. In this system configuration the matrices of stiffness $\mathbf{K}_{c}$ and of damping $\mathbf{B}_{c}$ of the linear connection can be calculated from the equation:

$$
\frac{\partial E_{p}^{(c)}}{\partial \mathbf{q}}+\frac{\partial D^{(c)}}{\partial \dot{\mathbf{q}}}=\mathbf{K}_{c} \mathbf{q}(t)+\mathbf{B}_{c} \dot{\mathbf{q}}(t)+\mathbf{f}^{I}(t),
$$

where $E_{p}^{(c)}$ - potential energy, $D^{(c)}$ - dissipative function of the connection, $\mathbf{f}^{I}(t)$ - vector of internal kinematic excitation in the connection.

\subsubsection{Modelling of the meshing}

Force acting between the gear teeth (Fig 3) can be determined from the relation:

$$
\mathbf{f}_{z}=\mathbf{B}_{z}(t) \dot{\mathbf{q}}_{z}(t)+\mathbf{K}_{z}(t) \mathbf{q}_{z}(t),
$$

where $\mathbf{K}_{z}(t)$ - matrix of meshing stiffness, $\mathbf{B}_{z}(t)-$ matrix of meshing damping, $\mathbf{f}_{z}$-vector of generalised forces acting between gear teeth.

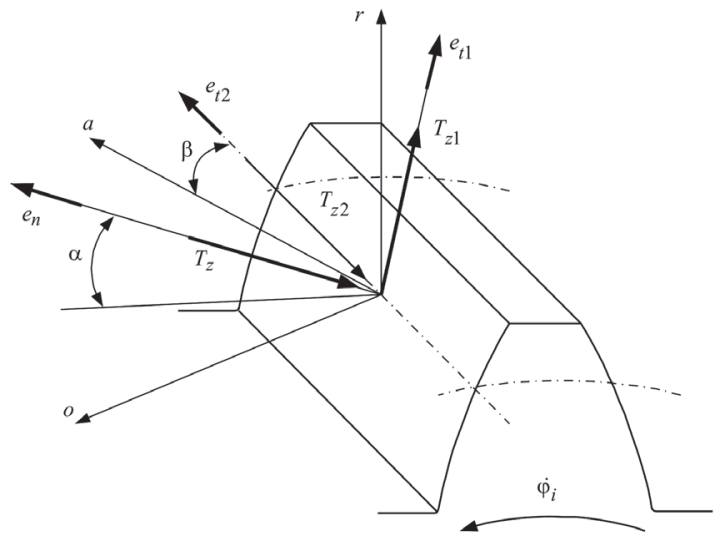

Fig 3. Forces acting on a gear tooth reduced to a meshing pole [13]

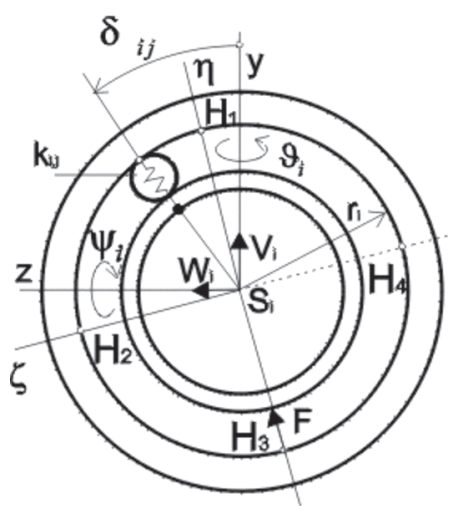

Interaction between teeth runs along the line of contact and the force acting between gear teeth can therefore be described by means of the following relation:

$$
N_{z}=k_{z}\left(d_{i}-d_{j}\right) e_{n}
$$

where $d_{i}, d_{j}$ are vectors of the displacement of contact points within the meshing pole resulting from angular and translational displacement of gears, whereas $e_{n}$ en is the versor of the axis orthogonal to the contact plane in this point, $k_{z}$ is the coefficient of stiffness of the meshing reduced to the contact line.

\subsubsection{Modelling of supports}

In toothed gears the form of support used most often is the rolling bearing (Fig 4). In the most common gear designs the flexibility of bearings plays a relatively minor role in the dynamics of gear trains. Likewise, the interaction of variable stiffness of meshing and nonlinearities occurring in a bearing have no large significance. In intricate dynamic models the stiffness of bearings should be calculated and entered into the numerical computation [14] of the model.

Force in the bearing can be determined from the relation:

$$
\mathbf{f}_{B}=\mathbf{B}_{B}(t) \dot{\mathbf{q}}_{B}(t)+\mathbf{K}_{B}(t) \mathbf{q}_{B}(t),
$$

where $\mathbf{K}_{B}(t)$ - matrix of stiffness in the bearing, $\mathbf{B}_{B}(t)$ - matrix of damping in the bearing, $\mathbf{f}_{B}$ column matrix of generalised forces in the bearing.

Radial contact of rolling elements between inner and outer ring of the bearing and axial contact between the casing and the shaft may be averaged to point $H_{j}$ with radius $r_{i}$ remote from centre $S_{i}$ in node ,i”, its position is described by angle $d_{i, j}$ measured from y axis. $H_{j}$ point is intermediate between node points $\overline{H_{j}}, \overline{H_{j}}$ in casing, the actual displacement between shaft and casing in a radial direction is:

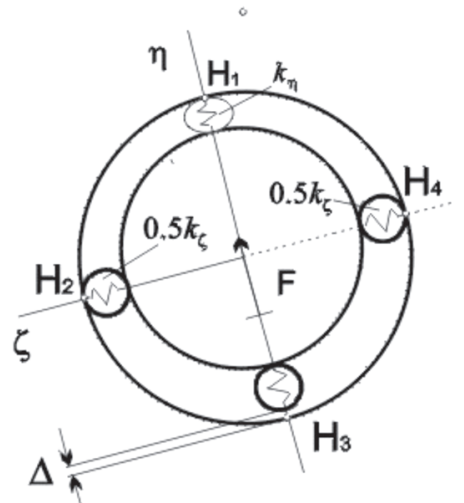

Fig 4. Model of a rolling bearing [15] 
$d_{i, j}=\left(v_{i}-\frac{\overline{y_{j}}}{2}-\frac{\overline{y_{j}}}{2}\right) \cos \delta_{i, j}+\left(w_{i}-\frac{\overline{z_{j}}}{2}-\frac{\overline{z_{j}}}{2}\right) \sin \delta_{i, j}$

and in an axial direction:

$d_{i, j a x}=u_{i}+r_{i} \sin \delta_{i, j} \vartheta_{i}-r_{i} \cos \delta_{i, j} \psi_{i}-\frac{\overline{x_{j}}}{2}-\frac{\overline{\overline{x_{j}}}}{2}$,

where $u_{i}, v_{i}, \psi_{i}, \omega_{i}, \vartheta_{i} \mathrm{Ji}$ is the deflection of , il" node on shaft in relation to bearing, whereas $\overline{x_{j}, \overline{x_{j}}}, \overline{y_{j}}, \overline{y_{j}}, \overline{z_{j}}, \overline{z_{j}}$ are the displacements of the node $\overline{H_{j}}$ and $\overline{H_{j}}$ of the casing along $\mathrm{x}, \mathrm{y}, \mathrm{z}$ axes of the system.

\section{Condensed model composed of subsystems}

A mathematical model of the entire system composed of $N_{p}$ subsystems interconnected by links can be written down as:

$\mathbf{M}_{j} \ddot{\mathbf{q}}_{j}(t)+\left(\mathbf{B}_{j}+\omega_{j, 0} \mathbf{G}_{j}\right) \dot{\mathbf{q}}_{j}(t)+\mathbf{K}_{\mathbf{j}} \mathbf{q}_{j}(t)=\mathbf{f}_{j}^{c}+\mathbf{f}_{j}(t)$,

$j=1,2, \ldots, N_{p}$,

where the ,j" index indicates a successive subsystem. In the case of housing the angular velocity is zero $\omega_{j, 0}$.

The effect of the preceding subsystem on the current subsystem $j$ " is expressed by means of couplings, represented by vector $\mathbf{f}_{j}^{c}$. This methodology is applied also for shafts where we most often have meshing and bearing constraints. In the configuration system defined by the global vector of generalised equations $\mathbf{q}(t)=\left[\mathbf{q}_{j}(t)\right]$ for $n=\sum n_{j}$, and the global vector $\mathbf{f}_{c}=\left[\mathbf{f}_{j}^{c}\right]$, of internal linear forces in couplings can be expressed as:

$$
\begin{aligned}
\mathbf{f}_{c} & =-\frac{\partial E_{p}^{(C)}}{\partial \mathbf{q}}-\frac{\partial D^{(C)}}{\partial \dot{\mathbf{q}}}= \\
& -\left(\mathbf{B}_{G}+\mathbf{B}_{B}\right) \dot{\mathbf{q}}(t)-\left(\mathbf{K}_{G}+\mathbf{K}_{B}\right) \mathbf{q}(t)+\mathbf{f}_{G}(t),
\end{aligned}
$$

where $\mathbf{K}_{G}, \mathbf{B}_{G}$ are the matrices of stiffness and of damping of all couplings in meshing, and, $\mathbf{K}_{B}, \mathbf{B}_{B}$ in all bearing couplings.

Modal properties of isolated and undamped subsystems in a certain range of frequencies are represented by a diagonal spectral submatrix ${ }^{m} \boldsymbol{\Lambda}_{j}$ of rank $m_{j}$ and by a rectangular modal submatrix ${ }^{m} \mathbf{V}_{j}$ of type $\left[n_{j}, m_{j}\right]$, where $m_{j}$ is the number of free vibration frequencies of subsystem , $j$ " within the given range of frequencies. Matrix ${ }^{m} \mathbf{V}_{j}$ is composed of $m_{j}$ master mode shapes of normalised free vibrations, respective free vibrations of , $;$ ” subsystem in the frequency range. Both matrices satisfy $\mathrm{M}$-orthogonal and K-orthogonal condition.

${ }^{m} \mathbf{V}_{j}^{T} \mathbf{M}_{j}{ }^{m} \mathbf{V}_{j}=\mathbf{I}, \quad{ }^{m} \mathbf{V}_{j}^{T} \mathbf{K}_{j}{ }^{m} \mathbf{V}_{j}={ }^{m} \boldsymbol{\Lambda}_{j}, j=1,2, \ldots, N_{p}$.

Modal transformation is:

$\mathbf{q}(t)=\mathbf{V} \mathbf{x}(t)$.

For single subsystems $\mathbf{q}_{j}(t)={ }^{m} \mathbf{V}_{j}{ }^{m} \mathbf{x}_{j}(t)$, $j=1,2, \ldots$. Then the model can written down in the form:

$\ddot{\mathbf{x}}(t)+\left(\mathbf{B}+\omega_{0} \mathbf{G}\right) \dot{\mathbf{x}}(t)+\boldsymbol{\Lambda} \mathbf{x}(t)=\mathbf{V}^{T}\left[\mathbf{f}_{C}+\mathbf{f}(t)\right]$,

where

$$
\begin{gathered}
\mathbf{B}=\operatorname{diag}\left({ }^{m} \mathbf{V}_{j}^{T} \mathbf{B}_{j}{ }^{m} \mathbf{V}_{j}\right), \\
\mathbf{G}=\operatorname{diag}\left(\frac{\omega_{j, 0}}{\omega_{0}}{ }^{m} \mathbf{V}_{j}^{T} \mathbf{G}_{j}{ }^{m} \mathbf{V}_{j}\right)
\end{gathered}
$$

are diagonal block matrices of rank $m=\sum m_{j}$, and

$$
\begin{aligned}
& \boldsymbol{\Lambda}=\operatorname{diag}\left({ }^{m} \boldsymbol{\Lambda}_{j}\right), \boldsymbol{V}=\operatorname{diag}\left({ }^{m} \boldsymbol{V}_{j}\right), \\
& \boldsymbol{x}(t)=\left[{ }^{m} \boldsymbol{x}_{j}(t)\right] \in R^{m}, \boldsymbol{f}(t)=\left[\boldsymbol{f}_{j}(t)\right] \in R^{n} .
\end{aligned}
$$

By eliminating vector $\mathbf{f}_{c}$ from the model (15) using the relation (12) we obtain a condensed model of the system with a reduced number of degrees of freedom $m$

$$
\begin{aligned}
& \ddot{\mathbf{x}}(t)+\left[\mathbf{B}+\omega_{0} \mathbf{G}+\mathbf{V}^{T}\left(\mathbf{B}_{G}+\mathbf{B}_{B}\right) \mathbf{V}\right] \dot{\mathbf{x}}(t)+ \\
& {\left[\Lambda+\mathbf{V}^{T}\left(\mathbf{K}_{G}+\mathbf{K}_{B}\right) \mathbf{V}\right] \mathbf{x}(t)=\mathbf{V}^{T}\left[\mathbf{f}_{G}(t)+\mathbf{f}(t)\right]}
\end{aligned}
$$

Dynamic behaviour of the model (11) for the given range of frequencies corresponds to original behaviour of the uncondensed model. Between vector $\boldsymbol{x}(\mathrm{t})$ of dimension $m$ of coordinates of condensed model and $\mathbf{q}(t)=\left[\mathbf{q}_{j}(t)\right]$ of dimension $n$ of generalised coordinates of model (11) there is a transformation relation (14). Using this transformation $\boldsymbol{q}(t)=\boldsymbol{V} \boldsymbol{x}(t)$ we revert to the base of original generalised coordinates.

\section{Computational model}

In order to determine the effect of housing flexibility on dynamic phenomena occurring in toothed gears numerical simulation was carried out for a onestage straight-toothed gear train driven by an asynchronous motor (Fig 5). 


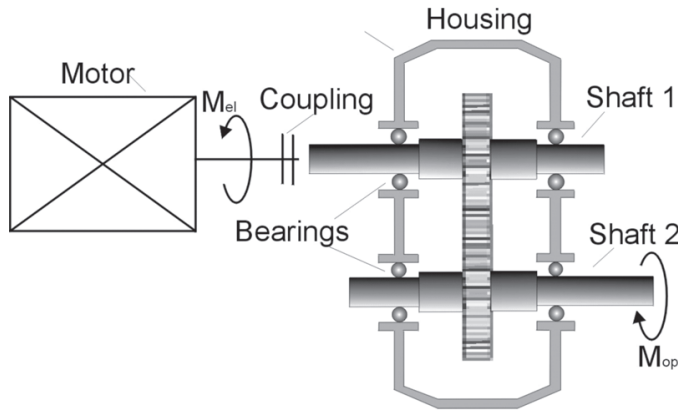

Fig 5. Model of a one-stage toothed gear train

Such an approach will enable assessing the effect of housing stiffness on dynamic phenomena occurring in drive systems which may be important for the modelling of electromechanical high power transmission systems.

The proposed model of the toothed gear includes housing fixed to a rigid substrate. The gear is driven by an asynchronous electric motor the electromagnetic moment of which is transferred by means of a coupling onto a one-stage straight-toothed gear train. Toothed gears are set in the middles of the shafts, the latter being elastically supported at mounting points of rolling bearings. The housing is coupled with the gear train using a rolling bearing model. Each bearing seat has 4 elastic damping elements of variable stiffness assigned to it.

Resisting moment $\mathrm{M}_{\mathrm{op}}$. is applied to the gear train output. The meshing is described by time-dependent stiffness $k_{z}(t)$ with account taken of play between teeth $l_{z}$ and of damping between teeth $b_{z}$.

The model of the gear train set in the housing is broken down into 3 subsystems - individual shafts with gears and the housing which interact through forces between teeth and forces in the bearing (Fig 6). These are internal forces in general terms. The hybrid method described in section 6 was applied to model the shafts with gears, while the model of the gear train housing was created with Ansys. Files with the reduced matrices of inertia and stiffness were exported using the internal APDL language of Ansys.

Two models were created to determine the effect of housing flexibility on the drive system:

- model of a toothed gear train without housing,

- model of a toothed gear train with housing.

\section{Numerical computations}

Numerical computations of the model of the gear train with the housing included the determination of displacement and speed variations in time, dynamic interactions in kinematic pairs and electromagnetic moment of the motor, both during start up as well as during steady operation. Computations were carried out on a PC computer using Ansys, MatLab and other proprietary software written in Clanguage. The Runge - Kuttay - Gill method was used to solve ordinary differential equations which describe the motion of the model gear train with the housing.

Numerical computation results are presented in Table and Fig 7-10.

The first step consisted of creating a simple model of a gear train (32 degrees of freedom) and determining its characteristics. Subsequently simulation was performed for a model of a one-stage gear train with housing. To decrease the number of degrees of freedom of the drive system the number of degrees of freedom in the housing model was reduced to 60 and then the entire system had 82 degrees of freedom. A simulation was performed on the adopted system. The node displacements obtained enabled the assessment of the effect of housing flexibility on the dynamics of the drive system.

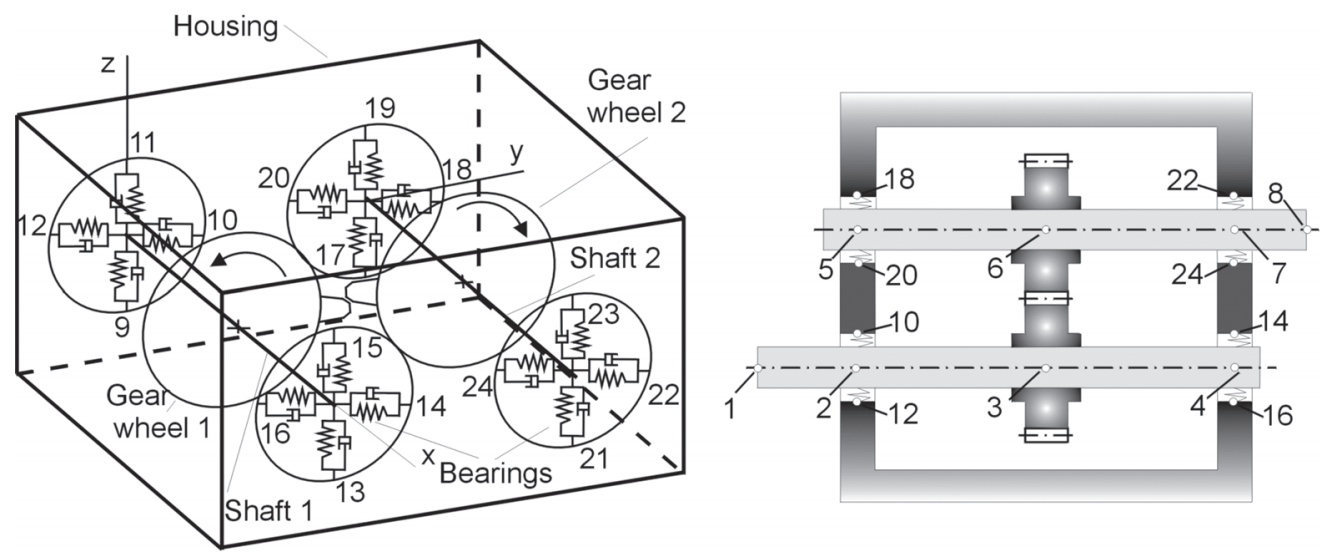

Fig 6. Model of a toothed gear train with housing [15] 
Frequency of free vibrations

\begin{tabular}{|c|c|c|}
\hline \multirow{2}{*}{$\begin{array}{c}\text { No of free } \\
\text { frequency }\end{array}$} & Gear train without housing & Gear train with housing \\
\cline { 2 - 3 } & 0 & 0 \\
\hline 1 & 481,08 & 413,22 \\
\hline 2 & 581,14 & 472,84 \\
\hline 3 & 581,14 & 559,13 \\
\hline 4 & 581,14 & 563,63 \\
\hline 5 & 658,92 & 568,56 \\
\hline 6 & 809,64 & 652,00 \\
\hline 7 & 2147,08 & 739,98 \\
\hline 8 & 2147,08 & 809,64 \\
\hline 9 & 2147,08 & 863,69 \\
\hline 10 & 2147,08 & 913,12 \\
\hline 11 & & \\
\hline
\end{tabular}

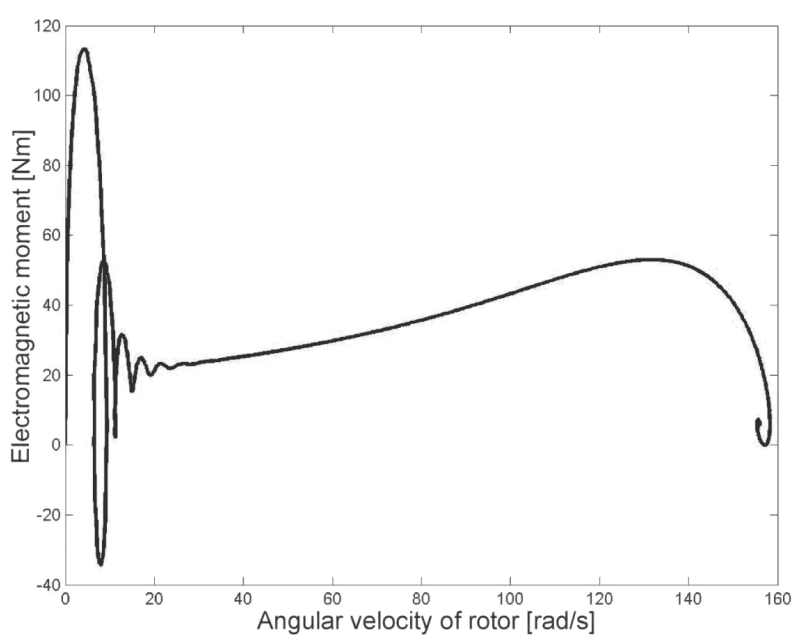

Fig 7. Electromagnetic moment vs. angular velocity of rotor

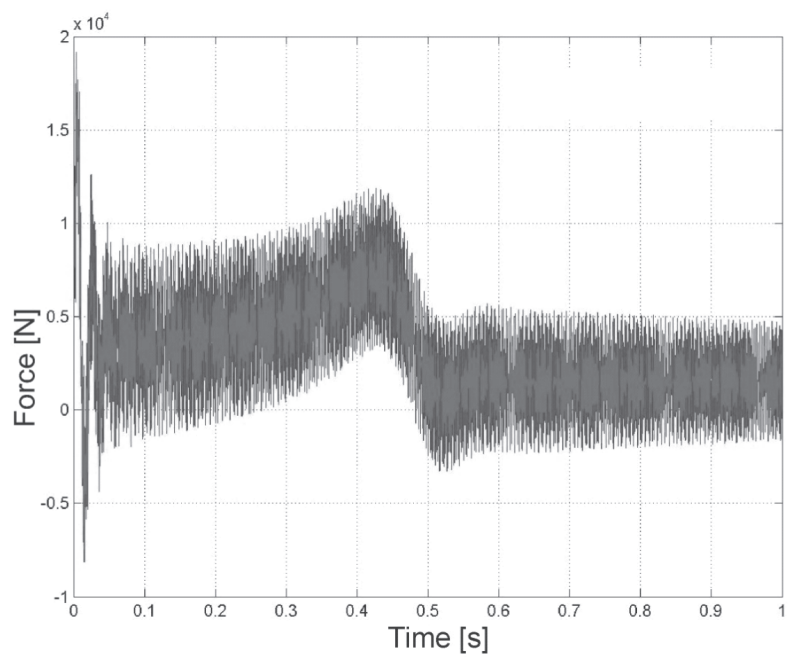

Fig 8. Force between teeth vs. time in a system with a housing

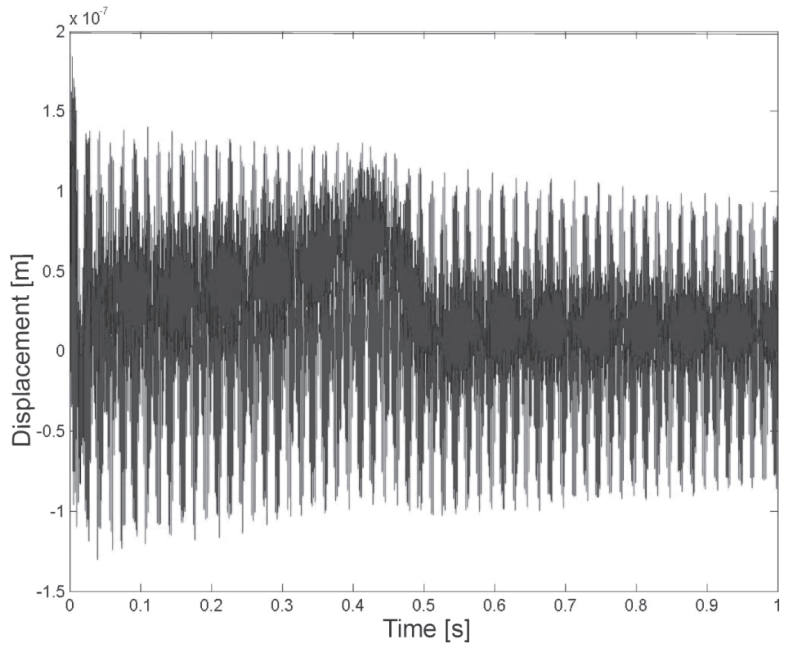

Fig 9. Displacement of node 2 on the shaft and of corresponding node 9 on the housing along $\mathrm{z}$ axis vs. time

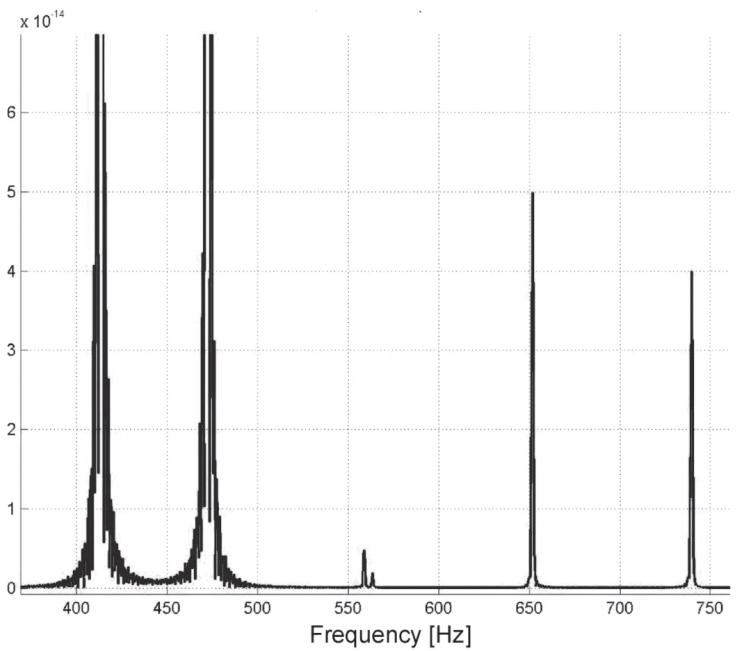

Fig 10. Identified frequency spectra in node 9 (on the housing) of the system with a housing along $\mathrm{z}$ axis corresponding to free vibration frequencies $\mathrm{f} 2=413.22 \mathrm{~Hz}$, $\mathrm{f} 3=472.84 \mathrm{~Hz}, \mathrm{f} 4=559.13 \mathrm{~Hz}, \mathrm{f} 5=568.56 \mathrm{~Hz}, \mathrm{f} 7=652 \mathrm{~Hz}$ and $\mathrm{f} 8=739.98 \mathrm{~Hz}$ 


\section{Conclusions}

This paper presented a method of modelling electromechanical systems as a drive system of high power machines. Each model developed consists of a mechanical part and an electrical part coupled by means of angular velocity of the rotor of the drive motor and by an electromechanical moment.

The model of the mechanical system is described using the finite element method which takes into account elements with continuously and discretely distributed mass. This model forms a kinematic chain with kinematic pairs which take into account nonlinearities ensuing from physical and kinematic links in bearings, meshing and couplings. This model also takes into account gyroscopic effects in rotating elements.

A model of the housing of a gear train was developed with the use of the finite element method. Subsequently the number of its degrees of freedom was reduced which resulted in considerable shortening of the time taken by numeric computations of the entire drive system coupled with the housing.

The numerical computations performed for such a model of an electromechanical system indicate that in transient states (start-up, sudden change in load, etc.) generalised forces of the magnitude much higher than nominal are generated in kinematic pairs of the system. The comparison of the spectra obtained indicates that when the housing is not taken into account in the computation of the dynamics of systems consisting of toothed gear trains, the vibration frequencies of the housing do not occur. When the gear train operates near the resonance frequency, these frequencies can have a decisive effect on dynamic forces in kinematic pairs of the drive system.

The developed dynamic model of the drive system and computation algorithms are of general nature and can be applied in the analysis of any electromechanical system with the possibility of imposing arbitrary initial-boundary conditions and various failure states.

The obtained amplitude-frequency characteristics enable the determination of parameters for diagnostic assessment and the data required for strength and fatigue calculations of the components of a drive system.

Undesired phenomena in coupled electromechanical systems occur particularly often in transient and resonant states. Therefore complex dynamic models comprising mechanical and electromagnetic parts are created to enable the analysis of such systems. In the case of large and complex electromechanical systems the condensation of the system is an advisable procedure. The reduction of the number of degrees of freedom of the model enables faster static and dynamic analysis.
The developed computer software can be applied in the design and construction process of drive systems in machines when the effect of housing stiffness on dynamic characteristics of the system has to be taken into account. The analysis of the investigations performed and the evaluation of numerical computation results obtained indicate that further research is required in the field of:

- optimal selection of dynamic features of the model system,

- inclusion in the mechanical system model the effects of dissipation energy, particularly in the meshing,

- further development of electromechanical models that take into account nonlinear phenomena in the meshing,

- further development of algorithms of optimising dynamic features of drive systems of machines.

\section{References}

1. Mężyk, A. Dynamics at electromechanical transmissions. Doctoral Dissertation (Dynamika elektromechanicznych układów napędowych. Rozprawa Doktorska), Gliwice, 1994 (in Polish).

2. Mężyk A. Optimization of dynamic parameters at machine transmissions. In: Collections Scientific Reports at Department at Applied Mechanics (Zeszyty Naukowe Politechniki Slaskiej, Mechanika), Vol 139, z. 139, Gliwice, 2002 (in Polish).

3. Gąsiorek, D.; Duda, S.; Kciuk, S.; Guzik, A. Verification of the FEM model of the frame of narrow arm for the longwall shearer prototype by using classical modal analysis. In: Collections Scientific Reports at Department at Applied Mechanics, Vol 139. Zescyty Naukowe Katedry Mechaniki Stosowanej, Vol 21, p. 57-60, Gliwice, 2003 (in Polish).

4. Kudla, J.; Mężyk, A.; Świtoński, E. Modelling of nonlinear vibrations of longwall shearerdriving system. In: Proceedings of International Scientific Conference, Ostrava, 12-15.09, 1995.

5. Jedlicka, Z. Simulation and the seismic analysis at the streched shell systems with dependent frequency parameters. Summary of Doctoral Dissertation (Modelovani a seizmicka analyza rozsahlnych teleso-potrubnich systemu s frekvencne zavislymi parametry. Autoreferat do Rozprawy Doktorskiej). Pilzn, 2003 (in Chezch).

6. Bollinger, J. G.; Harker, R. J. Instability Potential of High Speed Gearing. The Journal of the Industrial Mathematics Society, 1967, Vol 117.

7. David, J., W.; Mitchell, L. D. Linear Dynamic Coupling in Geared Rotor Systems. Journal of Vibration, Acoustic, Stress and Reliability in Design. Transactions of ASME, Vol 108, 1986.

8. Oehlmann, H.; Brie, D.; Tomczak, M.; Richard, A. A method for analysing gearbox faults using time-frequency representations. Mechanical System and Signal 
Procesing, 1997, 11(4), p. 529-545.

9. Özgüven, H. N.; Houser, D. R. Mathematical Models Used in Gear Dynamics-a Review. Journal of Sound and Vibration, 1988, Vol 121(3).

10. Van Dooren R. Comments On "Non-Linear Dynamics of Gear-Pair Systems With Periodic Stiffness And Backlash". Journal of Sound and Vibration, Vol 244, No 5, July 2001, p. 899-903.

11. Tuplin, W. A. Dynamic Loads on Gear Teeth. Machine Design, 1953, Vol 25.

12. Seireg, A.; Houser, D. R. Evaluation of Dynamic Factors for Spur and Helical Gears. Transcation of the ASME. Journal of Engineering for Industry, May 1970.

13. Rak, Z. Simulation of bending and rotational oscilations at electromechanical transmissions. Doctoral Dissertation (Modelowanie drgań giętno-skrętnych elektromechanicznych ukladów napedowych. Rozprawa Doktorska). Gliwice, 1995 (in Polish).

14. Nelson, H. D.; McVaugh, J. M. The Dynamics of Rotor-Bearing Systems Using Finite Elements. Journal of Engineering for Industry, May 1976.

15. Zeman, V.; Kovar, L. Modeling of dynamic properies of shaft and rotating system. Engineering Mechanics, Vol 1, Issue 6, 1999, p. 45-61. 